\title{
Controlling the Self-Injection Threshold in Laser Wakefield Accelerators
}

\author{
S. Kuschel,${ }^{1,2}$ M. B. Schwab, ${ }^{1,2}$ M. Yeung, ${ }^{1}$ D. Hollatz, ${ }^{2}$ A. Seidel, ${ }^{2}$ W. Ziegler, ${ }^{1,2}$ \\ A. Sävert, ${ }^{1,2}$ M. C. Kaluza, ${ }^{1,2}$ and M. Zepf ${ }^{1,2,3}$ \\ ${ }^{1}$ Helmholtz Insitute Jena, Fröbelstieg 3, 07743 Jena, Germany \\ ${ }^{2}$ Institute of Optics and Quantumelectronics, University of Jena, Max-Wien-Platz 1, 07743 Jena, Germany \\ ${ }^{3}$ School of Mathematics and Physics, Queens University Belfast, BT7 1NN, United Kingdom
}

(Received 21 May 2018; published 8 October 2018)

\begin{abstract}
Controlling the parameters of a laser plasma accelerated electron beam is a topic of intense research with a particular focus placed on controlling the injection phase of electrons into the accelerating structure from the background plasma. An essential prerequisite for high-quality beams is dark-current free acceleration (i.e., no electrons accelerated beyond those deliberately injected). We show that small-scale density ripples in the background plasma are sufficient to cause the uncontrolled (self-)injection of electrons. Such ripples can be as short as $\sim 50 \mu \mathrm{m}$ and can therefore not be resolved by standard interferometry. Background free injection with substantially improved beam characteristics (divergence and pointing) is demonstrated in a gas cell designed for a controlled gas flow. The results are supported by an analytical theory as well as 3D particle in cell simulations.
\end{abstract}

DOI: 10.1103/PhysRevLett.121.154801

The intense laser fields achievable with the current technology allow the generation of large-amplitude plasma wakefields with associated strong longitudinal electric fields in low-density plasmas. These wake structures propagate at velocities close to the speed of light, allowing the acceleration of electrons by so-called laser wakefield acceleration (LWFA) [1,2]. Such accelerators have accelerated 100-pC electron pulses to energies in excess of $1 \mathrm{GeV}$ over an acceleration length of a few centimeters only. These electron beams provide the basis for a number of further uses, such as compact sources of x rays, gamma rays, or positrons [3]. The concurrent availability of highly relativistic electrons and a high-intensity laser has opened up the field of high-intensity and nonlinear QED to experimental investigation, allowing fundamental theoretical concepts to be tested in the laboratory [4-8]. Furthermore, free-electron lasers are most demanding in terms of electron beam quality $[9,10]$.

Although the maximum energy of the accelerated electrons has increased significantly over the past decade, the energy bandwidth, charge, and divergence are crucial parameters that are still the subject of intensive research. In order to control those parameters, the injection process into the plasma wake has to be well controlled. Rather than injecting electron pulses from an externally prepared

Published by the American Physical Society under the terms of the Creative Commons Attribution 4.0 International license. Further distribution of this work must maintain attribution to the author(s) and the published article's title, journal citation, and DOI. electron source, the typical approach is to trap ("inject") and accelerate electrons from the background plasma in the wakefield. Various injection schemes as self-injection [11], ionization injection [12-15], colliding pulse injection [16], or shock front injection [17] have been studied, and others such as two-color injection [18] have been proposed.

Many experiments to date rely on self-injection to generate an electron beam (here we shall refer to any mechanism not separately controlled as "self-injection," but it is typically attributed to wave breaking [19-21]). However, the prerequisite for the ability to shape the electron bunch's spectrum or to fully explore another injection mechanism is the ability to drive a plasma wake without having any injection at all. Otherwise, every measurement of a distinct injection process will, at best, result in the measurement of this process in combination with uncontrolled self-injection. This is particularly critical at the moderately nonlinear regime, where good estimates apply [22] and self-injection is generally encountered in experiments. Understanding the microscopic causes of selfinjection is clearly central to designing stable laser-driven plasma wake accelerators. While there have been investigations of the self-injection threshold previously, no detailed experimental investigation of the microscopic process has been published to date.

In this Letter, we identify the small-scale density fluctuations as the primary cause of self-injection for experiments with moderate laser field strength $a_{0}$. We developed a gas cell and show that we can excite a strong plasma wave without self-injection of any electrons in pure helium. Electrons can be trapped and accelerated in this wave by ionization injection (adding 5\% nitrogen to the 
helium) and keeping all other laser and plasma parameters constant. We compare the performance of this gas cell with a gas-jet target that exhibits self-injection in a wide parameter range.

The experiment was conducted at the JETI-40 laser facility. The $10 \mathrm{~Hz}$ JETI Ti:sapphire laser delivers up to $E=650 \mathrm{~mJ}$ pulses at $\lambda_{0}=800 \mathrm{~nm}$ with $\tau=28 \mathrm{fs}$ duration on target. The beam was focused by an $f / 12$ off-axis parabolic mirror to a spot diameter of $\approx 12 \mu \mathrm{m}$ (FWHM) containing about $30 \%$ of the energy. This results in a normalized vector field amplitude of $a_{0}=$ $\sqrt{\lambda^{2} /\left[\mu \mathrm{m}^{2}\right] I /\left(1.37 \times 10^{18}\left[\mathrm{~W} / \mathrm{cm}^{2}\right]\right)} \approx 2.2$.

In the first part of the experiment, the laser focus was aligned to the front of a 2-mm-long gas jet, $0.75 \mathrm{~mm}$ above the nozzle. We will refer to this scenario as the "gas jet."

In the second scenario, we replaced the gas jet by our newly developed gas cell of the same length. The laser focus was aligned to its entrance aperture, and the gas inlets inside the gas cell were specifically located off-axis as well as tilted sideways to avoid any direct gas flow towards the laser axis. The cell had an inner diameter of $10 \mathrm{~mm}$, and the gas flow was controlled by a pulsed valve with typically $50 \mathrm{~ms}$ opening time with the laser pulse arriving $3 \mathrm{~ms}$ before the end of the fill period.

A Lanex scintillation screen allowed both beam profiles and spectra (by inserting a dipole magnet) to be recorded.

Both targets were adjusted to a length of $2 \mathrm{~mm}$. The gas jet and cell had been characterized [23] interferometrically with respect to the backing pressure, allowing the density to be scanned over the same range for a direct comparison. The plasma wake was imaged directly using a few-cycle probe pulse $\left(\tau_{\text {probe }}=6 \mathrm{fs}\right)$ [24]. Figure 1 shows a comparison of typical plasma wake images for these two cases. Looking at the wakefield close to the front of the interaction, we can see that the structure is essentially identical within the optical resolution $(\approx 1.5 \mu \mathrm{m})$ of the imaging, with the closely matched shape, modulation depth, and wakefield period visible, indicating similar conditions inside the plasma. The average plasma density $n_{p}=(1.2 \pm 0.2) \times 10^{19} / \mathrm{cm}^{3}$ was identical in both cases as measured by interferometry. The significant difference is the uniformity of the wakefield period across the image: While the cell displays a highly periodic structure [Fig. 1(a)], the periodicity is seen to vary along the plasma wake in the case of the gas jet [Fig. 1(b)]. The periodicity of the plasma wake provides a measurement of the local plasma wavelength and, therefore, of the local plasma density along the path of laser propagation. The observed fluctuations of the periodicity are consequently caused by plasma density variations or ripples in the gas jet, which are absent in the gas cell. Please note that the measurement of the local plasma wavelength along the laser axis provides unprecedented spatial resolution, and these density ripples cannot be resolved by interferometry techniques which
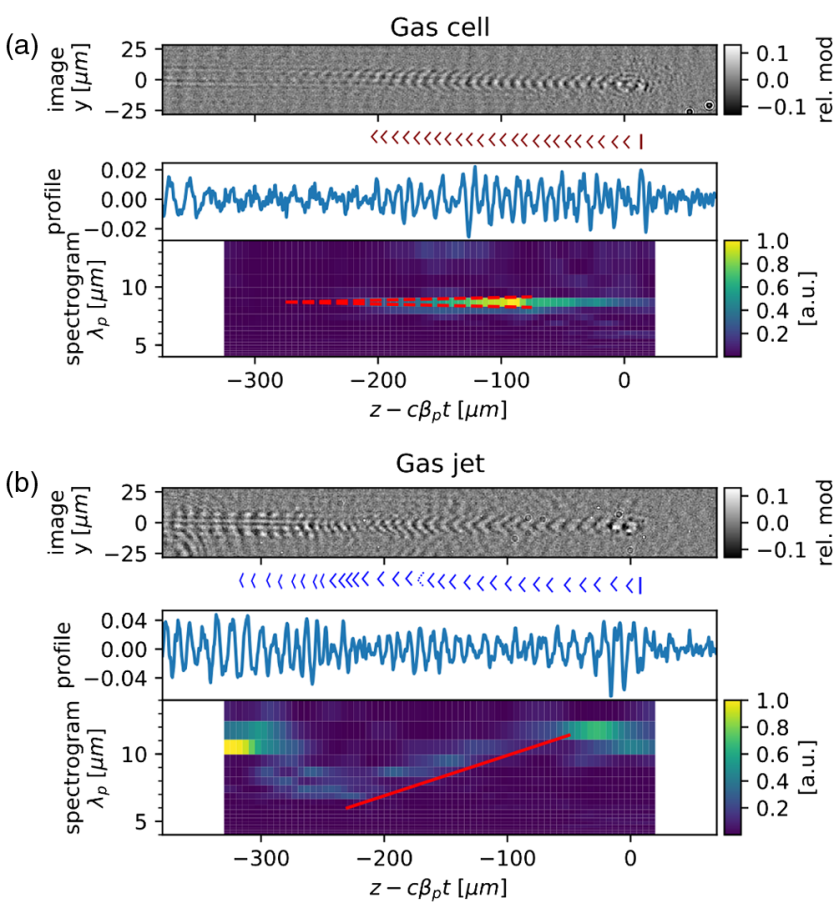

FIG. 1. Analysis of two typical shadowgrams of the wake recorded by transverse probing for the gas cell (a) and the gas jet (b) at the same nominal conditions of $n_{p}=$ $(1.2 \pm 0.2) \times 10^{19} / \mathrm{cm}^{3}$. The laser pulse propagates from left to right and is located at $z-c \beta_{p} t=0 \mu \mathrm{m}$ in the image. Both images were taken within the first $0.5 \mathrm{~mm}$ of each target. Experimental shadowgrams are shown in the top followed by a plot highlighting the phase fronts for clarity. A profile along $z$ within $y= \pm 10 \mu \mathrm{m}$ is shown in the center. The analysis of this profile (spectrogram) is in the bottom panel. In the case of the gas cell (a), $\lambda_{p}$ shows no dependence on the longitudinal position. The slope of the two red dashed lines corresponds to a gradient of $\partial_{z} \lambda_{p}= \pm 0.25 \%$. In the case of the gas jet (b), $\lambda_{p}$ shows a strong dependence on the longitudinal position. The slope of the red line corresponds to a gradient of $\partial_{z} \lambda_{p}=3 \%$. The change of the local plasma wavelength provides a measurement of the small-scale plasma density gradient (a single density ripple) over the range of only a few $\lambda_{p}$.

measure the phase shift along a line of sight over the entire width of the plasma.

Figure 2 compares the accelerated charge for three different target configurations: the gas jet using $\mathrm{He}$ and the gas cell using pure $\mathrm{He}$ as well as a mixture of $\mathrm{He}$ and $\mathrm{N}_{2}$. For the gas jet, electron beams are visible for densities greater than $0.8 \times 10^{19} / \mathrm{cm}^{3}$. Beam profiles for consecutive shots at the optimal and most stable conditions [found at a density of $(1.2 \pm 0.2) \times 10^{19} / \mathrm{cm}^{3}$ for both targets] are shown in Fig. 3. Note that the electron beam profile generated by the gas jet is not a single, smooth distribution but instead is highly structured, and the brightest point of the beam exhibits significant pointing fluctuations $(12 \mathrm{mrad}$ rms). The gas cell performs very differently compared to the gas jet in several respects: For a pure He fill, no electron 


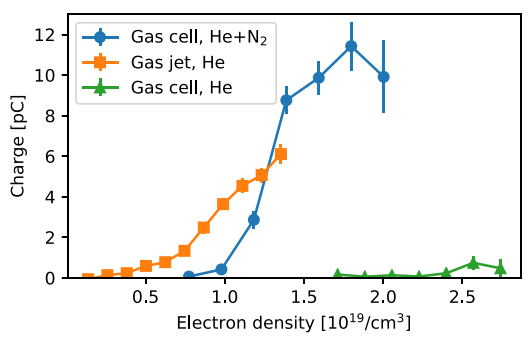

FIG. 2. Accelerated charge depending on the electron density for different target configurations. The error bars indicate the standard error for each data point. The onset of the electron injection for the gas jet was $n_{e} \approx 0.8 \times 10^{19} / \mathrm{cm}^{3}$ (below which the electron beam was highly unstable), corresponding to $P / P_{c}=1.8$. No electron beam was observed in the helium-filled cell up to $n_{e}=2.75 \times 10^{19} / \mathrm{cm}^{3}$, corresponding to $P / P_{c}=5.5$.

injection is observed for the full density range, showing clearly that the mechanism leading to self-injection in the jet is suppressed for the cell. When the gas is changed to a $\mathrm{He}(95 \%)-\mathrm{N}_{2}(5 \%)$ mixture to allow the injection of electrons via the ionization of the nitrogen $K$-shell electrons by the drive laser, an electron beam with a comparable maximum energy (up to $150 \mathrm{MeV}$ ) is observed. Under these conditions, electrons are accelerated for a wide range of densities (Fig. 2) in a highly stable beam in terms of beam structure, pointing, and divergence (Fig. 3). No difference was discernible in the probe images for the two gas mixtures in the cell.

In the case of the gas jet, electron beams were observed for plasma densities $n_{e} \gtrsim 0.8 \times 10^{19} / \mathrm{cm}^{3}$. Using the gas cell with pure He, however, even for the highest density of $n_{e}=2.75 \times 10^{19} / \mathrm{cm}^{3}$, no self-injection was observed in the experiment using the gas cell.

By comparing the results with and without nitrogen, we can ascribe the observed electron signal from the gas cell as being purely due to ionization injection. The beam profile consists of a single, smooth beam with a low divergence [density dependent rising to $2.2 \mathrm{mrad}$ at the peak beam

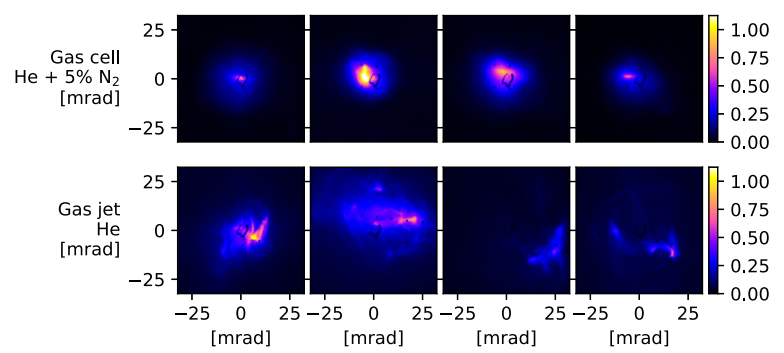

FIG. 3. Consecutive $e$-beam profiles at optimal conditions $\left[n_{e}=(1.2 \pm 0.2) \times 10^{19} / \mathrm{cm}^{3}\right]$ for both targets. Gas cell (top) beam profiles are stable and almost unstructured with a pointing variation of $4 \mathrm{mrad} \mathrm{rms}$. Under these conditions, the gas jet (bottom) results in a strongly structured beam with significant pointing fluctuations (13 mrad rms). The dark ring in the center is a reference mark on the scintillation screen. brightness at $\left.n_{e}=(1.2 \pm 0.2) \times 10^{19} / \mathrm{cm}^{3}\right]$ and stable pointing (4 mrad rms). Thus, in the case of the gas cell, we have a self-injection free operating mode driven by a laser with vacuum $a_{0}=2.2$, providing the basis for the full control of the electron beam phase space through external injection.

The remarkable difference between the cell and the jet in terms of self-injection and beam properties can be understood when we consider the electron trapping mechanism which typically underlies self-injection. Trapping of background electrons occurs when some of the plasma electrons overcome the phase velocity of the plasma wake $c \beta_{p}$, allowing them to slip into the accelerating phase of the plasma wake (this process is frequently also referred to as wave breaking). The phase velocity of the wake $c \beta_{p}$ is reduced to values less than $c$ in a uniform plasma by dispersion (reducing the group velocity of the laser) and socalled pulse "etching" (where the front of the pulse is preferentially absorbed $[22,25])$. In nonuniform plasmas, the phase velocity is additionally reduced by density downramps [26]. They cause an increase of the plasma wavelength, increasing during the passage through the density transition, resulting in a locally reduced velocity of the phase fronts of the plasma wake. With $\zeta$ as the distance between the driving laser pulse and the phase front of the plasma wake, the reduction purely due to the downramp is described by [26]

$$
\beta_{p}^{\text {down }}=\frac{1}{1+\frac{\zeta}{k_{p}} \frac{d k_{p}}{d z}} .
$$

We approximated this expression using $\Delta \lambda_{p} \ll \lambda_{p}$ and simplified substituting $n=\omega m_{e} \epsilon_{0}$ and $\omega=2 \pi c / \lambda_{p}$ :

$$
\beta_{p}^{\text {down }} \approx 1-\frac{\zeta}{2 n} \frac{d n}{d z}=1-\frac{\zeta}{\lambda_{p}} \frac{\partial \lambda_{p}}{\partial z} .
$$

Consequently, a sufficiently rapid increase of the plasma wavelength decreases $\beta_{p}$ and can induce electron trapping [20]. Assuming $\zeta \approx \lambda_{p}$ for the first wakefield period (which is actually a lower limit [27]), we can therefore construe $\partial_{z} \lambda_{p}$ as the additional term contributing to the reduction of $\beta_{p}$. Expressing this effect in terms of the plasma wavelength allows us to evaluate the relative influence on $\beta_{p}$ directly from the recorded probe images. Analyzing the plasma wake fluctuations from Fig. 1, the different contributions to $\beta_{p}$ evaluate to

$$
\beta_{p}=1-\underbrace{\frac{1}{2} \frac{\omega_{p}^{2}}{\omega^{2}}}_{0.3 \%}-\underbrace{\frac{\omega_{p}^{2}}{\omega^{2}}}_{0.6 \%}-\underbrace{\frac{\partial \lambda_{p}}{\partial z}}_{\substack{\text { jel: } \sim 3 \% \% \\ \text { cell: }<0.25 \%}} .
$$

Dispersion and etching (first two terms; see [22,25]) sum up to $0.9 \%$ (at $n_{p}=1 \times 10^{19} / \mathrm{cm}^{3}$ ), and the density ripples 


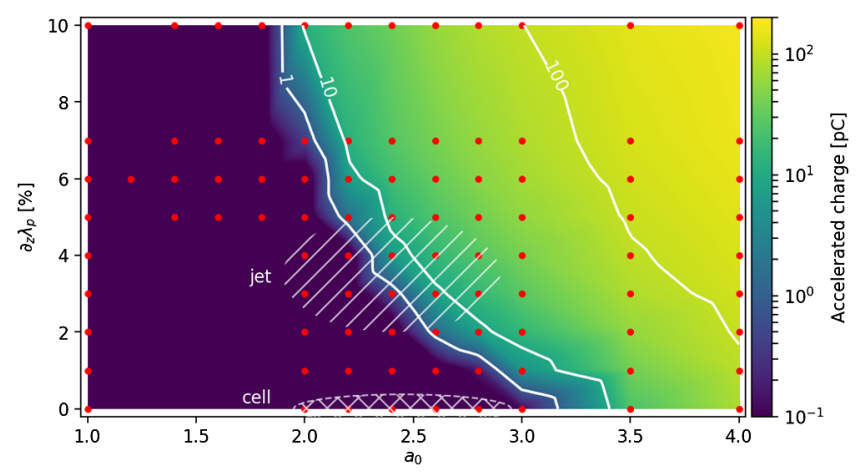

FIG. 4. Accelerated charge in pC depending on initial $a_{0}$ of the laser and the downramp gradient $\partial_{z} \lambda_{p}$. Simulations were performed for every red dot, while the color scale and contour lines are interpolated. The sudden onset for wave breaking is indicated by the onset of the color scale at $0.1 \mathrm{pC}$, and contour lines at 1,10 , and $100 \mathrm{pC}$ are shown. The typical operating range of the gas jet and gas cell are also indicated separating the different regimes.

present in the gas jet are identified as the leading contribution of $\sim 3 \%$ [Fig. 1(b)]. The ripples show up randomly during the experiment, and also some extreme cases of up to $\sim 10 \%$ were measured repeatedly. The gas cell, in contrast, provides a smooth plasma density with gradients smaller than the sensitivity of the imaging, which is $\left[\left(\partial \lambda_{p}\right) /(\partial z)\right]<0.25 \% \quad$ [Fig. 1(a)]. Experimentally and analytically, it is therefore clear that the uncontrolled injection we observe in the gas jet is caused by the density ripples.

To gain quantitative insight on the effect of a single density ripple on the injection threshold, we performed 3D particle in cell (PIC) simulations using the code ЕPOCH [28] studying how a downramp can trigger self-injection (Fig. 4). A laser pulse with variable $a_{0}$ is injected into the simulation box of $60 \mu \mathrm{m}$ length and $\left(30+5 a_{0}\right) \mu \mathrm{m}$ in both transverse directions. The focus plane is set to $z=0 \mu \mathrm{m}$, which is at the center of a $100-\mu \mathrm{m}$-long linear ramp to the maximum density. After $55 \mu \mathrm{m}$ of propagation through the plasma, a stable plasma wake has formed, while the self-modulation of the laser pulse due to the plasma may still be neglected. At $z=105 \mu \mathrm{m}$, a downramp of $30 \mu \mathrm{m}$ length (representing a single density ripple) with constant $\partial_{z} \lambda_{p}$ is encountered by the laser pulse. The density on the center of the ramp was always set to $n_{p}=1 \times 10^{19} / \mathrm{cm}^{3}$, matching the experimental conditions. Figure 4 shows the injected charge depending on the (vacuum) $a_{0}$ of the laser pulse and the gradient of the plasma density in units of $\partial_{z} \lambda_{p}$. It can be clearly seen that the simulation reproduces the results from the experiments: For $a_{0}=2.2$, no injection occurs in the simulation for $\partial_{z} \lambda_{p}<4 \%$. Even if self-focusing of the laser pulse possibly increases the $a_{0}$ inside the plasma over longer propagation distances, an $a_{0}>3$ would be required for self-injection to occur in the absence of density ripples. For the measured gradients of $\partial_{z} \lambda_{p} \approx 3 \%$ in the gas jet, an $a_{0} \gtrsim 2.4$ is already sufficient to trigger electron injection. Of course, even stronger density gradients allow wave breaking at even smaller laser intensities. Identical to the experiment, no trapping of background electrons is observed for conditions matching the gas cell $\left(a_{0}=2.2\right.$, $\left.\partial_{z} \lambda<0.5 \%\right)$. These findings are in agreement with recent simulations [29], furthermore suggesting the creation of attosecond electron bunches in this regime [30].

The simulations are in excellent agreement with our experiment and the analytical considerations above and provide a quantitative scaling on how density ripples and the concomitant reduction of $\beta_{p}$ reduce the self-injection threshold well below the threshold value of $a_{0}$ observed for uniform plasmas. In agreement with the simulations, no trapping of background electrons is observed for our experimental conditions $\left(a_{0}=2.2\right)$ using the gas cell.

Expressing the injection threshold in terms of the ratio of the laser power to the critical power for self-focusing $P / P_{c}$ allows a comparison with published experiments. For our conditions, the threshold for self-injection in the gas jet is $P / P_{c}=1.8$. These values fit well with the range of thresholds published in gas jet experiments in Refs. [31,32] of $P / P_{c} \sim 2$ and $P / P_{c} \sim 3$, respectively. The range of threshold values then corresponds to small changes in the typical ripple amplitude found in different gas jet designs. The injection threshold for our gas cell with pure He shifts substantially to $P / P_{c} \sim 4.6$ for the observation of any accelerated charge and beyond $P / P_{c}>5.5$ for the observation of a well-defined electron beam, again in agreement with our simulations. Note that measured values of $P / P_{c}$ typically have absolute uncertainties of around $30 \%$ stemming from the difficulty in determining the laser power in the laser focus and the absolute density of the target. As our measurements have been carried out at the same laser system, it is safe to state that the gas cell raises the $P / P_{\text {crit }}$ injection threshold by a factor of 3 compared to the injection threshold of the gas jet.

With the picture of self-injection occurring at density ripples, the structured electron beam profiles and larger pointing fluctuations of the gas jet are also straightforward to explain: Since the self-injected electron beam consists of many separate injection events caused by recurring density ripples which are random in position along the laser axis and orientation to it, one expects to see an electron beam profile consisting of multiple beamlets, which is in agreement with the structured beam profiles observed using the gas jet (Fig. 3), while the single bright beam in the gas cell is consistent with a single injection mechanism and a stable wakefield. Our simulations also indicate further that the pointing of the laser and electrons is affected by density gradients that are not parallel to the laser axis.

The influence of density ripples on the scale of a few plasma wavelengths increases when the plasma density is decreased. Demanding that random density gradients should have a smaller influence than etching and dispersion 
combined translates to $\partial_{z} \lambda_{p}<\frac{3}{2}\left(n_{p} / n_{c}\right)$. This is equivalent to $\left[\left(\lambda_{p}\right) /\left(2 n_{p}\right)\right]\left[\left(\partial n_{p}\right) /(\partial z)\right]<\frac{3}{2}\left(n_{p} / n_{c}\right)$ and displays the unfavorable scaling if the plasma density $n_{p}$ is reduced. It is therefore of great importance to acknowledge this influence in future LWFA or PWFA devices operated at significantly lower plasma densities.

In conclusion, we have demonstrated that self-injection free acceleration is possible for a large range of densities for laser plasma interactions with $a_{0} \approx 2.2$, opening the path to LWFA beams with excellent beam quality and to background free acceleration. We have identified the primary microscopic mechanism leading to self-injection in the gas jet: Density ripples are the leading contribution to the reduction of the phase velocity of the wake $\beta_{p}$ and hence to self-injection. The 3D PIC simulations reproduce the strongly nonlinear self-injection process and the amount of accelerated charge for our parameters. Multiple injection events caused by the density ripples are also likely the cause for the structured beam profiles and pointing fluctuations observed using the gas jet. The appreciation that small-scale density fluctuations that randomly occur in gas flows can be the source of the accelerated electrons and their structured beam profiles is also crucial in interpreting many of the experimental results published to date and designing future LWFA or PWFA systems. Beyond the enhanced beam quality and stability, simulations suggest that this regime, newly accessed in our experiment, allows the creation of attosecond electron bunches [30], which may lead to the development of tabletop free-electron laser systems with attosecond duration.

B. Beleites and F. Ronneberger have contributed to this work by operating the JETI 40 laser facility. The authors gratefully acknowledge the computing time granted by the John von Neumann Institute for Computing (NIC) and provided on the supercomputer JURECA at Jülich Supercomputing Centre (JSC). The authors acknowledge the following open-source-projects: Numpy [33], Matplotlib [34], IPython [35], and postpic [36]. M. B. S., A. S., M. C. K., and M. Z. acknowledge support from the Bundesministerium für Bildung und Forschung (Grants No. 05K16SJB and No. 05K16SJC).

[1] W. Leemans, P. Volfbeyn, K. Guo, S. Chattopadhyay, C. Schroeder, B. Shadwick, P. Lee, J. Wurtele, and E. Esarey, Laser-driven plasma-based accelerators: Wakefield excitation, channel guiding, and laser triggered particle injection, Phys. Plasmas 5, 1615 (1998).

[2] S. P. Mangles et al., Laser-Wakefield Acceleration of Monoenergetic Electron Beams in the First Plasma-Wave Period, Phys. Rev. Lett. 96, 215001 (2006).

[3] G. Sarri et al., Generation of neutral and high-density electron-positron pair plasmas in the laboratory, Nat. Commun. 6, 6747 (2015).
[4] K. Poder et al., Evidence of Strong Radiation Reaction in the Field of an Ultra-Intense Laser, Phys. Rev. X 8, 031004 (2018).

[5] M. Marklund and P. K. Shukla, Nonlinear collective effects in photon-photon and photon-plasma interactions, Rev. Mod. Phys. 78, 591 (2006).

[6] N. V. Elkina, A. M. Fedotov, C. Herzing, and H. Ruhl, Improving the accuracy of simulation of radiation-reaction effects with implicit Runge-Kutta-Nyström methods, Phys. Rev. E 89, 053315 (2014).

[7] A. Di Piazza, C. Müller, K. Hatsagortsyan, and C. Keitel, Extremely high-intensity laser interactions with fundamental quantum systems, Rev. Mod. Phys. 84, 1177 (2012).

[8] D. G. Green and C. N. Harvey, Transverse Spreading of Electrons in High-Intensity Laser Fields, Phys. Rev. Lett. 112, 164801 (2014).

[9] F. Grüner et al., Design considerations for table-top, laserbased VUV and x-ray free electron lasers, Appl. Phys. B 86, 431 (2007).

[10] A. Bernhard et al., Progress on experiments towards LWFAdriven transverse gradient undulator-based FELs, Nucl. Instrum. Methods Phys. Res., Sect. A, DOI: 10.1016/ j.nima.2017.12.052 (2017).

[11] S. Kalmykov, S. A. Yi, V. Khudik, and G. Shvets, Electron Self-Injection and Trapping into an Evolving Plasma Bubble, Phys. Rev. Lett. 103, 135004 (2009).

[12] M. Chen, Z.-M. Sheng, Y.-Y. Ma, and J. Zhang, Electron injection and trapping in a laser wakefield by field ionization to high-charge states of gases, J. Appl. Phys. 99, 056109 (2006).

[13] A. Pak, K. A. Marsh, S. F. Martins, W. Lu, W. B. Mori, and C. Joshi, Injection and Trapping of Tunnel-Ionized Electrons into Laser-Produced Wakes, Phys. Rev. Lett. 104, 025003 (2010).

[14] C. McGuffey, A. G. R. Thomas, W. Schumaker, T. Matsuoka, V. Chvykov, F. J. Dollar, G. Kalintchenko, V. Yanovsky, A. Maksimchuk, K. Krushelnick, V. Y. Bychenkov, I. V. Glazyrin, and A. V. Karpeev, Ionization Induced Trapping in a Laser Wakefield Accelerator, Phys. Rev. Lett. 104, 025004 (2010).

[15] C. E. Clayton, J. E. Ralph, F. Albert, R. A. Fonseca, S. H. Glenzer, C. Joshi, W. Lu, K. A. Marsh, S. F. Martins, W. B. Mori, A. Pak, F. S. Tsung, B. B. Pollock, J. S. Ross, L. O. Silva, and D. H. Froula, Self-Guided Laser Wakefield Acceleration beyond $1 \mathrm{GeV}$ Using Ionization-Induced Injection, Phys. Rev. Lett. 105, 105003 (2010).

[16] H. Kotaki, I. Daito, M. Kando, Y. Hayashi, K. Kawase, T. Kameshima, Y. Fukuda, T. Homma, J. Ma, L.-M. Chen, T. Z. Esirkepov, A. S. Pirozhkov, J. K. Koga, A. Faenov, T. Pikuz, H. Kiriyama, H. Okada, T. Shimomura, Y. Nakai, M. Tanoue et al., Electron Optical Injection with Head-On and Countercrossing Colliding Laser Pulses, Phys. Rev. Lett. 103, 194803 (2009).

[17] A. Buck et al., Shock-Front Injector for High-Quality Laser-Plasma Acceleration, Phys. Rev. Lett. 110, 185006 (2013).

[18] L.-L. Yu, E. Esarey, C. B. Schroeder, J.-L. Vay, C. Benedetti, C. G. R. Geddes, M. Chen, and W. P. Leemans, Two-Color Laser-Ionization Injection, Phys. Rev. Lett. 112, 125001 (2014). 
[19] S. V. Bulanov, F. Pegoraro, A. M. Pukhov, and A. S. Sakharov, Transverse-Wake Wave Breaking, Phys. Rev. Lett. 78, 4205 (1997).

[20] S. Bulanov, N. Naumova, F. Pegoraro, and J. Sakai, Particle injection into the wave acceleration phase due to nonlinear wake wave breaking, Phys. Rev. E 58, R5257 (1998).

[21] P. Tomassini, M. Galimberti, A. Giulietti, D. Giulietti, L. A. Gizzi, L. Labate, and F. Pegoraro, Production of highquality electron beams in numerical experiments of laser wakefield acceleration with longitudinal wave breaking, Phys. Rev. Accel. Beams 6, 121301 (2003).

[22] W. Lu, M. Tzoufras, C. Joshi, F. Tsung, W. Mori, J. Vieira, R. Fonseca, and L. Silva, Generating multi-GeV electron bunches using single stage laser wakefield acceleration in a 3d nonlinear regime, Phys. Rev. Accel. Beams 10, 061301 (2007).

[23] B. Landgraf, M. Schnell, A. Sävert, M. C. Kaluza, and C. Spielmann, High resolution 3d gas-jet characterization, Rev. Sci. Instrum. 82, 083106 (2011).

[24] M. Schwab et al., Few-cycle optical probe-pulse for investigation of relativistic laser-plasma interactions, Appl. Phys. Lett. 103, 191118 (2013).

[25] C. D. Decker, W. B. Mori, K. Tzeng, and T. Katsouleas, The evolution of ultra-intense, short-pulse lasers in underdense plasmas, Phys. Plasmas 3, 2047 (1996).

[26] E. Esarey, C. B. Schroeder, and W. P. Leemans, Physics of laser-driven plasma-based electron accelerators, Rev. Mod. Phys. 81, 1229 (2009).

[27] A. Sävert et al., Direct Observation of the Injection Dynamics of a Laser Wakefield Accelerator Using FewFemtosecond Shadowgraphy, Phys. Rev. Lett. 115, 055002 (2015).

[28] T. D. Arber, K. Bennett, C. S. Brady, A. Lawrence-Douglas, M. G. Ramsay, N. J. Sircombe, P. Gillies, R. G. Evans,
H. Schmitz, A. R. Bell, and C. P. Ridgers, Contemporary particle-in-cell approach to laser-plasma modelling, Plasma Phys. Controlled Fusion 57, 113001 (2015).

[29] F. Massimo, A. Lifschitz, C. Thaury, and V. Malka, Numerical studies of density transition injection in laser wakefield acceleration, Plasma Phys. Controlled Fusion 59, 085004 (2017).

[30] M. P. Tooley, B. Ersfeld, S. R. Yoffe, A. Noble, E. Brunetti, Z. M. Sheng, M. R. Islam, and D. A. Jaroszynski, Towards Attosecond High-Energy Electron Bunches: Controlling Self-Injection in Laser-Wakefield Accelerators through Plasma-Density Modulation, Phys. Rev. Lett. 119, 044801 (2017).

[31] S. P. D. Mangles, G. Genoud, M. S. Bloom, M. Burza, Z. Najmudin, A. Persson, K. Svensson, A. G. R. Thomas, and C.-G. Wahlström, Self-injection threshold in self-guided laser wakefield accelerators, Phys. Rev. Accel. Beams 15, 011302 (2012).

[32] D. H. Froula, C. E. Clayton, T. Döppner, K. A. Marsh, C. P. J. Barty, L. Divol, R. A. Fonseca, S. H. Glenzer, C. Joshi, W. Lu, S. F. Martins, P. Michel, W. B. Mori, J. P. Palastro, B. B. Pollock, A. Pak, J. E. Ralph, J. S. Ross, C. W. Siders, L. O. Silva et al., Measurements of the Critical Power for Self-Injection of Electrons in a Laser Wakefield Accelerator, Phys. Rev. Lett. 103, 215006 (2009).

[33] S. v. d. Walt, S. C. Colbert, and G. Varoquaux, The numpy array: A structure for efficient numerical computation, Comput. Sci. Eng. 13, 22 (2011).

[34] J. D. Hunter, Matplotlib: A 2d graphics environment, Comput. Sci. Eng. 9, 90 (2007).

[35] F. Pérez and B. E. Granger, Ipython: A system for interactive scientific computing, Comput. Sci. Eng. 9, 21 (2007).

[36] S. Kuschel, postpic: The open-source particle in cell postprocessor, http://github.com/skuschel/postpic. 